\title{
Éthique et enseignement en Europe
}

Charles Ballarin, Eugénie Errea, Martine Souquès et Jocelyne Thébault

\section{(2) OpenEdition}

Journals

Édition électronique

URL : http://journals.openedition.org/ries/4156

DOI : $10.4000 /$ ries.4156

ISSN : 2261-4265

\section{Éditeur}

Centre international d'études pédagogiques

\section{Édition imprimée}

Date de publication : 5 mars 1995

Pagination : 55-65

ISSN : 1254-4590

Référence électronique

Charles Ballarin, Eugénie Errea, Martine Souquès et Jocelyne Thébault, «Éthique et enseignement en Europe », Revue internationale d'éducation de Sèvres [En ligne], 05 | 1995, mis en ligne le 16 mars 2015, consulté le 02 mai 2019. URL : http://journals.openedition.org/ries/4156 ; DOI : 10.4000/ries.4156

Ce document a été généré automatiquement le 2 mai 2019.

(c) Tous droits réservés 


\title{
Éthique et enseignement en Europe
}

\author{
Charles Ballarin, Eugénie Errea, Martine Souquès et Jocelyne Thébault
}

\section{NOTE DE L'AUTEUR}

Cet article présente le premier état des travaux d'un groupe de professeurs consultants auprès du département des systèmes éducatifs du Centre international d'études pédagogiques (CIEP) de Sèvres. L'ensemble des travaux donnera lieu à la rédaction d'un rapport.

1 Nous nous proposons d'étudier tout d'abord les termes employés dans différentes langues pour qualifier le domaine moral, selon qu'il s'agit de la réflexion sur la vie morale ou sur le comportement individuel. Puis nous nous interrogerons sur la mission que peut ou doit assumer l'école dans ce domaine. Enfin, nous tenterons de dresser un état des lieux dans les systèmes éducatifs étudiés.

\section{Comparaisons lexicales}

Entre la philosophie, qui prend pour objet l'étude des fondements de la morale, et le degré d'intériorisation qu'implique l'adhésion à un code de vie personnel, on peut identifier deux étapes : les systèmes de principes ou de règles de conduite et ce qui relève des comportements, de la science des jugements et des appréciations.

Dans toutes les langues étudiées le terme " éthique », etika en russe, ética en espagnol, etik en suédois, ethik en allemand, ethics en anglais, du grec ethos (coutume, usage, habitude), désigne le domaine de la philosophie consacré à la vie morale en général. En allemand comme en suédois, le terme désigne également l'apprentissage (par l'enseignement) de la morale. L'emploi du pluriel en anglais est à remarquer : il connote en effet, par rapport au singulier, une liberté de penser, d'envisager diverses hypothèses. 
Car en anglais, dans les cas, relativement rares, où l'on rencontre ce terme au singulier, c'est l'aspect codifié des systèmes de règles de conduite qui est mis en valeur, ainsi que l'idée que l'individu y adhère pour ainsi dire totalement. On parle donc de «l'éthique chrétienne ", de "l'éthique puritaine» ou de «l'éthique victorienne du travail» au singulier. Toutes ces éthiques constituent des codes, mais, dans l'usage, l'accent est mis sur l'adhésion personnelle. En revanche, l'emploi du même terme, pour désigner à la fois la théorie et le code, ne permet pas autant de subtilité dans l'usage.

5 C'est par rapport aux systèmes de principes et de règles de conduite que des divergences apparaissent dans les langues. En effet, en français, en suédois, comme en espagnol et en russe, le terme "morale », moraliska en suédois, moral en espagnol, moralj en russe, s'emploie aussi pour désigner « un ensemble de principes, de règles dont une personne s'inspire pour diriger sa conduite » (Boileau, 1694). Il apparaît cependant qu'en français le caractère normatif de toute morale est nettement plus marqué qu'en espagnol; en effet, la plupart des définitions données par le Grand Larousse de la langue française mettent l'accent sur cet aspect : dans la mesure où la morale désigne l'étude théorique, cette étude vise «à des conséquences normatives, des fins et des principes de l'action humaine " (Descartes, 1637). Il s'agit d'un ensemble de règles de conduite "admises inconditionnellement et considérées comme applicables à tous » (Pascal, 1662). L'unique marge de liberté, la seule souplesse admise est celle qui limite un tel système à une époque ou à un groupe d'hommes. Le suédois moraliska est un terme qui vient du français. En espagnol en revanche un terme différent, moralidad, apparaît pour désigner les «mœurs ».

6 Lorsqu'on passe du domaine théorique et des systèmes/codes au domaine plus mouvant de la morale pratique, deux niveaux se dégagent : celui du groupe et celui de l'individu. Pour ce qui concerne le groupe, la réflexion sur les conduites et les jugements est désignée en français par le terme "éthique ", ética en russe, etik en suédois, ética en espagnol. Ce terme est souvent précisé par des adjectifs et des préfixes tel «bio». En anglais en revanche, le pluriel ethics est employé systématiquement pour désigner l'ensemble de règles de conduite adopté par un groupe d'individu, par exemple dans le domaine professionnel. Traditionnellement on pense aux professions libérales lorsqu'on emploie ce terme, car chacune de ces professions initie ses futurs membres à la déontologie concernée. Dans tous les cas, une déontologie de ce type autorise à porter un regard extérieur sur les comportements : il s'agit de porter un jugement sur la conformité ou la non-conformité d'un comportement individuel à un code extérieur.

7 L'expression suédoise "valeurs éthiques", qui désigne ce qui devrait constituer une déontologie dans des domaines tels que la médecine, la bioéthique, l'informatique, voire les relations internationales, apparaît comme une manière très actuelle de désigner ce type de code. En effet, le terme "valeur ", dont le sens « caractère des choses consistant en ce qu'elles sont plus ou moins estimées ou désirées par un sujet ou plus ordinairement par un groupe de sujets déterminés » (Grand Larousse de la langue française) ne varie guère d'une langue à l'autre, introduit dans le domaine moral la notion de relativisme. Adopté par le positivisme, il met l'accent beaucoup plus nettement sur l'individu, que celui-ci ait adopté les valeurs d'un groupe ou d'une religion ou qu'il se soit forgé son propre code. De nos jours, il est souvent question des "valeurs" auxquelles adhère l'individu. Il peut aussi bien s'agir d'un code que d'un ensemble de valeurs relevant de différentes traditions. 
8 Lorsqu'il s'agit de morale personnelle nous trouvons des termes plus variés. Le terme russe nravstvienost qui vient du mot nrav (le caractère) est sans doute, avec le terme anglais principles (" an inward and personal law of right action»; " honourable character ») le terme le plus intériorisé. De manière générale, on rencontre deux termes, «principes » et « valeurs », selon que l'on insiste davantage sur l'intériorisation ou sur le fait de se mettre en conformité avec un groupe. En espagnol comme en anglais ou en suédois, on parle donc de "principes moraux» pour désigner une morale personnelle adoptée par l'individu qui la reconnaît comme le fondement de ses actes et tend à la suivre consciemment. Il s'agit d'un code intériorisé, d'un élément formateur de la personnalité, voire d'une expression de celle-ci.

9 Lorsque le domaine moral est évoqué dans les textes officiels, ce sont les termes « éthique », « morale», mais aussi «valeurs » qui sont employés. En ce qui concerne les deux premiers, "éthique " s'emploie pour décrire un enseignement (théorique ou pratique), alors que la formation aux jugements et aux choix est souvent décrite par le terme «morale»: la seule exception étant l'emploi de l'adjectif «moral» dans les programmes d'éducation civique en France, emploi comparable à celui de "vertus civiques" (civic virtues) aux États-Unis. Ce domaine n'est pas toujours mentionné explicitement dans les textes, même si tous les systèmes éducatifs dont il est question ici s'intéressent d'une manière ou d'une autre aux attitudes, sinon aux comportements des élèves. On a d'un côté les pays où il n'est guère question, dans les textes officiels, des mots "éthique » et "valeurs» et de l'autre, le Schleswig-Holstein ou l'Espagne où il est question à la fois de «l'éducation sociale », de « l'éducation morale » et de l'enseignement des savoir-faire, de certaines attitudes et de valeurs. Des pays comme l'Angleterre se situent entre les deux : on y trouve des termes tels que l'ethos de chaque établissement scolaire, la « pastorale » qu'il faut assurer auprès des élèves, la « dimension morale » de la vie à laquelle il faut qu'ils soient sensibilisés. En Suède, également, des questions éthiques sont inscrites aux programmes de nombreuses matières.

\section{Quelles missions pour l'école?}

Le débat actuel sur l'opportunité de confier aux systèmes éducatifs une mission éthique s'explique par quatre facteurs.

11 En premier lieu, on peut le considérer comme une version contemporaine du vieux débat sur l'équilibre que doit trouver l'école dans son double rôle de formation du futur adultecitoyen et d'épanouissement de l'individu. L'exemple le plus net de la subordination du deuxième objectif au premier est la France. Ainsi, le rapport annexé à la loi Jospin définit l'objectif du système éducatif comme la formation du futur adulte qui doit être capable de conduire sa vie personnelle, civique et professionnelle; il s'agit donc de former le futur citoyen et le futur travailleur. L'interprétation de l'adjectif « personnelle » est ambiguë, car, bien qu'il s'agisse de culture générale (il est fait mention du développement du goût de « créer, d'exercer des activités physiques, culturelles et artistiques »), l'accent est mis plus nettement sur le devoir qu'a l'école de prendre en charge le projet personnel de l'élève: "L'École doit permettre à l'élève d'acquérir un savoir et de construire sa personnalité par sa propre activité. La réalisation de cet objectif demande du temps : son utilisation optimale par l'élève est le problème essentiel de l'École... » ("Les missions et les objectifs fixés par la nation ", rapport annexé à la loi d'orientation sur l'éducation, juillet 1989). Si on prend le cas des États-Unis, la montée du personnalisme depuis les 
années 1960 met l'accent sur l'épanouissement de l'individu. Dans la plupart des pays cependant, l'orientation est moins nette que ne semblent l'indiquer ces deux exemples, soit parce qu'on adopte le principe de transversalité pour ce qui est du contenu et la discussion comme méthode de sensibilisation, soit parce que l'on ambitionne d'éveiller et de former le sens moral.

Le deuxième des facteurs qui suscitent le débat actuel est, dans certains pays, une conscience nouvelle de la nature pluriculturelle de la société, prise de conscience qui amène à poser des questions sur les valeurs que l'école doit véhiculer et enseigner. On pense naturellement aux États-Unis, bien qu'il s'agisse d'un cas complexe, dans la mesure où la reconnaissance des droits des minorités n'a rien modifié au traditionnel enseignement des valeurs civiques. La question se pose actuellement en Russie dans des termes analogues, quant à la relativité des valeurs, dans le contexte scolaire où diverses cultures sont mises sur un pied d'égalité. Mais dans ces deux pays, le débat sur l'éthique oppose différentes écoles de pensée et non différentes minorités : ainsi les partisans de la religion s'opposent aux humanistes. En Angleterre comme en Suède, en revanche, la reconnaissance du pluriculturalisme a conduit l'école à prendre en charge l'éducation à la tolérance raciale et l'enseignement des religions autres que chrétiennes.

Le troisième facteur, qui intervient semble-t-il, de manière réactive, et qui est donc moins lié à la situation actuelle, est une expérience historique d'intolérance, voire de persécution de la différence : différence idéologique, ethnique, religieuse. Ce facteur est intervenu en Allemagne où, après 1945, l'obsession étatique fut remplacée par un enseignement du civisme et de l'éthique qui met l'accent à la fois sur le développement du jugement moral individuel et sur la conscience politique. Il est également à l'origine de la montée de la conscience ethnique aux États-Unis dans le sillage du mouvement pour les droits civiques des années 1950 et 1960 et, après l'élection de John Fitzgerald Kennedy à la présidence, de l'importance nouvelle accordée aux origines ethniques des hommes politiques. Plus proche de nous est l'expérience récente de l'Espagne où, depuis l'adoption de la nouvelle constitution de 1978 et la reconnaissance de la liberté religieuse, débats, expériences et réforme s'élaborent dans le but de définir les visées et les méthodes d'un enseignement de l'éthique. En Russie enfin, la loi de la Fédération de Russie sur l'enseignement (juillet 1992) fixe comme objectifs l'éducation au civisme et le développement libre de la personnalité, les deux étant étroitement liés.

Les trois facteurs que nous venons d'examiner relèvent tous essentiellement du domaine du comportement public du citoyen, du travailleur, du consommateur, voire du critique de la société. Le quatrième facteur en revanche, concerne bien davantage les comportements privés et met donc en jeu les attitudes intimes. Il s'agit d'autre chose que d'encourager certains comportements censés caractériser le bon citoyen, comme c'est le cas aux États-Unis avec le service learning (initiation au bénévolat) qui est dans certains cas obligatoire, mais qui vise uniquement à former l'homme social. Ce qu'on peut qualifier de crise de nos sociétés - délinquance, drogue, problèmes liés à l'éclatement du noyau familial, influence du SIDA sur les comportements privés, comportements sexuels des jeunes - semble exiger une prise de position, voire une attitude éducatrice de la part de l'école. D'autre part, le sentiment est de plus en plus répandu que certains progrès scientifiques - dans le domaine de la bioéthique notamment - exigent que l'individu soit capable de prendre des décisions selon des critères moraux, en se référant, non pas à une morale qui sera enseignée une fois pour toutes, mais en adulte responsable, éclairé, c'està-dire en connaissance de cause. Cette préoccupation est tantôt justifiée par des 
arguments pragmatiques (coût pour la société de la délinquance), comme cela est le cas en Angleterre, tantôt pour des raisons plus idéologiques, parce que certains comportements sont susceptibles d'entraver l'épanouissement personnel ou bien sont, comme c'est le cas en Suède, contre "l'éthique qui a été transmise par la tradition chrétienne et par l'humanisme occidental ». Les conséquences pour l'école sont les mêmes: cette préoccupation se traduit par l'ambition de sensibiliser les jeunes, de développer leur sens moral par des objectifs transversaux, la méthode adoptée étant la discussion.

\section{L'éthique dans l'enseignement}

Dans les faits, l'éthique est enseignée à trois niveaux. L'Espagne et, dans une moindre mesure, l'Angleterre où les trois niveaux figurent dans le curriculum, sont des cas exemplaires. Dans la plupart des pays cependant, on choisit essentiellement entre un enseignement théorique ou une formation/sensibilisation aux comportements et aux choix.

On remarque deux conceptions différentes, mais souvent complémentaires sur le terrain, de la place et du rôle de l'éthique dans l'enseignement. D'une part, l'éthique est conçue comme une réflexion sur les comportements, sur les pratiques et sur les valeurs. L'éthique devient dans ce cas objet d'enseignement. D'autre part, l'éthique est conçue comme une sensibilisation aux valeurs ou encore comme une formation du caractère et du jugement moral. Il s'agit d'une éthique fondée sur la pratique : elle est présente de façon transversale dans le curriculum et de façon implicite dans la politique de l'école en matière de discipline, d'ambiance de l'établissement et des méthodes d'enseignement. Ces deux conceptions se traduisent, au niveau des curricula et des programmes, de trois manières différentes qui correspondent à trois niveaux d'enseignement.

17 En tant qu'objet d'enseignement, l'éthique figure dans les curricula sous deux formes, à savoir, en tant que matière autonome et sous la forme de l'éducation civique. En tant que matière autonome, elle est souvent proposée comme une alternative à la religion : ceci est le cas notamment en Allemagne où l'éthique n'est enseignée que dans les Länder où l'enseignement de la religion est obligatoire. En Espagne, l'éthique reste une alternative officielle à la religion dans les classes où la réforme n'est pas encore entrée en vigueur. Au fur et à mesure de la mise en application de celle-ci, l'alternative tend à disparaître. En Angleterre et en Suède, la connaissance des religions est obligatoire, aucune alternative n'est proposée. En Russie, la loi de programmation de juillet 1992 donne la possibilité aux établissements qui le désirent d'introduire l'éthique dans leur programme. Dans d'autres pays, aux États-Unis et France, ni l'une ni l'autre ne sont enseignées, encore qu'aux ÉtatsUnis plusieurs États, dont la Californie, ont réintroduit l'étude de la religion ces dernières années; cet enseignement a lieu dans le cadre des programmes de sciences sociales et vise des objectifs culturels.

Il est à noter que, dans certains Länder allemands, la philosophie, et non l'éthique, est proposée comme alternative à la religion. Parmi les Länder qui proposent un cours d'éthique, on note une orientation plus philosophique chez certains (la Sarre et la Hesse) tandis qu'en Bavière et en Rhénanie-Palatinat l'éthique repose sur certaines valeurs déterminées a priori, inscrites dans les constitutions des Länder, dans la Loi fondamentale de la République et dans la Déclaration universelle des droits de l'homme. Dans ces cas, il s'agit de rendre explicites des a priori qui sous-tendent l'enseignement direct de l'éthique. 
En Espagne, le programme de l'unité didactique « La vie morale et la réflexion éthique ", unité obligatoire enseignée en fin d'enseignement secondaire obligatoire, comprend une réflexion sur l'aspect théorique de l'éthique (le caractère historique et universel des valeurs morales; normes éthiques et pluralité morale en démocratie; les principales théories éthiques). Le curriculum anglais, tout en le subordonnant à l'apprentissage pratique, reconnaît le rôle de la raison dans la formation morale.

L'éducation civique est conçue de deux manières différentes. En Allemagne, il s'agit d'informer sur les droits, les devoirs et les institutions et de former le futur citoyen aux attitudes démocratiques, y compris à la critique. En France, depuis 1985, une dimension morale apparaît clairement dans les instructions officielles pour l'école élémentaire: «L'éducation civique apprend à l'enfant qu'il ne vit pas seul, qu'il procède d'une histoire, qu'il a des droits reconnus mais aussi des devoirs. Éminemment morale, l'éducation civique développe l'honnêteté, le courage, le refus des racismes, l'amour de la République » (Instructions officielles, 1985).

Cette dimension morale s'efface progressivement des programmes de l'école élémentaire. $\mathrm{Au}$ cours préparatoire, l'accent est mis sur « l'apprentissage des règles fondamentales de la vie en société - règles d'hygiène, de sécurité, de tenue, respect du matériel scolaire et des équipements collectifs, respect de soi et des autres, reconnaissance des droits d'autrui, de l'égalité des races et des sexes, de la dignité de la personne» (I.O., 1985). Il s'agit également de développer certaines qualités plus intériorisées : sens de l'effort et du travail bien fait, goût de l'autonomie et des responsabilités. Bien que la République soit déjà présente par ses symboles au cours préparatoire, c'est au cours élémentaire qu'intervient la justification de ces règles morales qui n'est autre que l'idée de la République elle-même. On présentera ainsi aux enfants, après les notions de personne, de propriété (le mien, le tien, le nôtre) et de contrat, la devise républicaine « liberté, égalité, fraternité », la patrie, unité et identité nationale, le droit de vote. Le couronnement du programme intervient au cours moyen durant lequel sont introduites la Déclaration des droits de l'homme et du citoyen de 1789 et la Déclaration universelle des droits de l'homme de 1948. Ce sont donc des notions politico-civiques qui justifient ou fondent les vertus morales. La morale apparaît comme la base de l'éducation civique, ce sur quoi l'ensemble s'édifie mais de telle sorte que, une fois la base acquise, il ne soit plus nécessaire d'y revenir. Une telle interprétation est confirmée par la lecture des instructions pour le premier cycle de l'enseignement secondaire. La dimension morale n'est plus mentionnée ; il s'agit à ce stade d'éclairer l'élève sur les vertus démocratiques, celles qui sont exigées d'un citoyen : «L'éducation civique suppose la compréhension des règles de la vie démocratique et de leurs fondements, la connaissance des institutions et de leurs racines historiques, la réflexion sur les conditions et les moyens du respect de l'homme et de ses droits dans le monde d'aujourd'hui : tolérance et solidarité, refus des racismes, volonté de vivre ensemble en démocratie. Elle met les élèves en mesure de répondre à leur propre exigence de liberté et de justice et de faire face, de manière responsable, aux problèmes et aux défis de notre temps » (I.O., 1985). Une partie importante du programme est consacrée à la connaissance des institutions départements, régions, Constitution de la $\mathrm{V}^{\mathrm{e}}$ République, rôle de l'État - mais aussi à une réflexion sur les droits et les libertés, le point culminant en fin de troisième étant les valeurs de la démocratie.

21 L'importance accordée à la formation du citoyen, fondée sur l'exercice de la raison et la connaissance d'une certaine culture, se poursuit dans le deuxième cycle : ainsi les mots 
importants dans les programmes de seconde sont « éducation » et « éducation civique ». En histoire, il est question du "développement de l'esprit critique» et de «l'apprentissage de la liberté »; en sciences économiques et sociales de "se préparer à travailler et à se comporter en adultes - producteurs, consommateurs mais aussi en citoyens - plus lucides, plus libres, plus conscients en même temps de leurs responsabilités ». Le mot « éthique » n'apparaît qu'en biologie.

Il ressort de l'examen de cet aspect du curriculum français qu'il est avant tout question de la formation aux comportements publics, soit de citoyen, soit de membre d'un groupe. En ceci, l'éducation civique en France ressemble à ce qui se passe aux États-Unis, où l'accent est mis sur la vie communautaire, sur le refus de la violence, sur certains comportements - refus du mensonge, respect d'autrui, etc. - parce qu'ils nuisent à la vie en société. Aux États-Unis cependant, ces valeurs communautaires sont transmises de manière indirecte ; elles ne font pas l'objet d'un enseignement direct. Pour ce qui relève de l'instruction civique à proprement parler, l'approche est différente: on s'appuie sur des exemples historiques, on prête serment quotidiennement devant le drapeau - mais l'objectif est le même. On assiste à l'heure actuelle, en France, à une réorientation de l'éducation civique. Au-delà de la connaissance des institutions démocratiques, son objectif est «l'acquisition d'une morale de la responsabilité dans différents domaines de la vie sociale et personnelle : environnement, santé, justice, etc. » (Un nouveau contrat pour l'école : rentrée 1994). Ce nouvel objectif devrait être atteint dans le cadre d'un enseignement spécifique.

À un deuxième niveau, l'éthique est enseignée de manière transversale, chaque discipline et chaque professeur ayant un rôle à jouer. Cette approche est privilégiée par la Suède et l'Angleterre ainsi qu'en Espagne où elle complète l'enseignement direct. En Suède, le rapport Skola för bildning (Une école pour la formation, 1992), qui inspira les nouveaux programmes de l'école obligatoire publiés en février 1994, évoque l'actualité des questions éthiques dans les domaines médical (biogénétique, échographie, chirurgie de la transplantation), informatique (problématique du secret de l'intégrité de la personne) et international (identité, réfugiés, ressources et environnement). Le rôle de l'école est d'expliquer et d'ancrer solidement les valeurs éthiques afin de bien préparer les élèves pour l'avenir : les élèves doivent être capables de réfléchir et de discuter des questions existentielles et éthiques. L'éthique apparaît dans les programmes d'histoire, de sciences sociales, de géographie, de sciences et sciences naturelles, de suédois et d'histoire de l'art. En Angleterre, les questions éthiques sont évoquées de manière analogue. Un des objectifs du curriculum national est de préparer les élèves aux choix de la vie adulte. Le curriculum est un véhicule pour promouvoir le développement spirituel, moral, culturel, intellectuel et physique des élèves de l'établissement et de la société. Au niveau du curriculum ces préoccupations se traduisent par les thèmes transversaux que toute discipline doit véhiculer (éducation à la santé, informatique, éducation politique, éducation économique, problèmes liés au monde du travail, orientation professionnelle, égalité des chances, des races et des sexes). Une grande importance est accordée à la discussion informée des motivations et des choix tant dans le domaine des rapports avec autrui (par le biais des textes littéraires, par exemple) qu'en science ou en géographie.

De même, en Espagne, l'unité didactique "La vie morale et la réflexion éthique " comprend une sensibilisation aux questions éthiques contemporaines (problèmes moraux dans la société contemporaine, projets éthiques contemporains tels l'écologie, l'autorité et sa légitimation, la religion comme fait individuel et social). 
26 En outre, en Espagne, les attitudes, normes et valeurs mises en œuvre dans chacun des champs disciplinaires sont définies officiellement au même titre que les concepts, les procédés et les méthodes d'apprentissage. Si on compare la langue maternelle et la littérature avec les langues étrangères, la manière dont les attitudes préconisées traduisent certaines valeurs apparaît clairement.

27 Exemple du champ disciplinaire langue maternelle et littérature Thème : utilisations et formes de la communication orale qu'on peut désigner par « valeur de la communication ». "Attitudes à adopter: intérêt et respect pour les opinions d'autrui, intérêt et respect pour les diversités linguistiques, les variantes idiomatiques, etc. » (El curriculo de la enseñanza secundaria obligatoria). Les valeurs mises en évidence sont bien: l'intérêt pour l'autre et le respect des différences. Les attitudes à développer consistent à prêter attention à celui qui parle, à ne pas l'interrompre, etc.

28 Exemple du champ disciplinaire langues étrangères

Thème : aspects socioculturels. Dans l'apprentissage de la langue étrangère étudiée, il s'agit de développer la curiosité et le respect des façons de vivre différentes, de mettre en valeur l'enrichissement personnel que suppose la relation à des personnes de culture différente et d'encourager des comportements pouvant faciliter les relations de convivialité.

29 Enfin, l'importance accordée dans les programmes aux attitudes à mettre en œuvre par les enseignants et à encourager chez les élèves traduit l'ambition d'agir sur les comportements de manière indirecte, par imprégnation, par l'ambiance dans laquelle se déroule l'enseignement. En Angleterre et de manière moins explicite en Allemagne, cette ambition se traduit par les efforts accomplis pour faire de l'école un lieu d'apprentissage de la vie en société, et le véhicule privilégié de l'éducation aux comportements. La vie en société se prépare par la manière dont se conçoit la vie de la classe, et par la participation éventuelle des élèves à la vie de l'établissement. En Angleterre, cet aspect de la mission de l'école est résumée par l'expression «information et occasions » de faire preuve d'initiative, de choisir en connaissance de cause, d'assumer des responsabilités et de diriger les autres.

30 L'ambiance générale des établissements, la façon dont l'institution réagit face à la violence, à la délinquance et aux conflits de toute sorte, ainsi qu'aux paroles ou aux gestes discriminatoires (racistes, sexistes), - ce qui est résumé par le mot ethos - relève de l'éducation morale. Ce que tentent de faire les écoles américaines, en refusant la violence par exemple, est systématisé et codifié en Angleterre. Ainsi, chaque établissement est tenu de définir, par la discussion entre enseignants, élèves, parents d'élèves et administration, une politique concernant les paroles racistes (attitudes, moyens de résoudre les conflits, sanctions éventuelles). Cette politique doit être définie dans un document écrit tenu à la disposition de tous. Les enseignants et l'institution ont l'obligation de se montrer solidaires d'un collègue victime de menaces ou d'une agression. En un mot, pour tout ce qui concerne la discipline et le respect le plus strict du principe d'égalité, l'institution est tenue de réagir d'une manière qui renforce dans l'esprit de tous certaines valeurs (tolérance, égalité, justice) considérées comme fondamentales. Il est à remarquer que, dans son discours du 9 mai 1994 à la Maison de la chimie, le ministre français de l'éducation, François Bayrou, a évoqué des mesures allant dans ce sens pour lutter contre la violence à l'école : soutien des enseignants qui rencontrent une crise, qui subissent une agression; mise en place d'instances plus souples que le conseil de 
discipline pour tenter de régler les conflits en amont ; adoption d'une loi claire, connue de tous avec des sanctions transparentes et effectivement appliquées.

31 À l'heure actuelle donc, le rôle qu'il convient de faire jouer à l'école dans la formation morale des jeunes fait l'objet d'une réflexion ou d'une réorientation des programmes ou de la pédagogie. En Russie et aux États-Unis, on en est au stade du débat au sein de l'opinion publique, mais en Suède, en Espagne et en Angleterre, on procède déjà à la mise en œuvre de réformes tendant à placer cette formation au centre du travail de l'école. En France, cette préoccupation s'est traduite par la modification des objectifs de l'éducation civique.

Pour ce qui est de l'approche pédagogique dans ce domaine, la tendance est nette selon laquelle toute tentative d'enseigner des valeurs, voire une morale, est abandonnée en faveur d'une sensibilisation à la dimension éthique/morale de la vie, sensibilisation qui se fait de manière moins directive - c'est-à-dire en adoptant le principe de la transversalité mais aussi plus complète. Cette réorientation va de pair avec une conscience plus nette du rôle que joue l'institution, qui exprime et transmet des valeurs, par la manière dont elle fonctionne, voire par son organisation.

La citation suivante décrit clairement la mission confiée à l'école dans les pays qui ont mené à terme la réflexion sur l'éthique : «La communauté scolaire, tout comme la vie de famille, est le cadre où ont lieu l'apprentissage et la mise en pratique de valeurs morales, et ceci de plusieurs façons. En premier lieu, dans ce contexte, sont mis en contact des actes moraux et les principes qui les sous-tendent. Si l'on veut que les élèves comprennent des notions telles que l'équité et la justice, il faut que les adultes à qui ils ont affaire y adhèrent et les appliquent. En deuxième lieu, l'école est un lieu où se déroulent des expériences qui contribuent à former et à tester les principes moraux et à modifier les attitudes. L'école est un lieu privilégié où les élèves peuvent apprendre à accepter l'idée que l'on doit tenir compte du point de vue et des réactions d'autrui, qu'un équilibre doit être trouvé entre les intérêts de l'établissement et ceux des individus, et qu'il est possible et désirable de résoudre les conflits en ayant recours à la raison. Mis à part cet apprentissage, il est cependant nécessaire que les élèves commencent à fonder leur pratique morale sur la raison. L'enseignement direct de la morale ne doit pas être banni de l'école, mais il est nécessaire d'aider les élèves, en particulier les plus grands, à raisonner sur les valeurs; la justesse des jugements moraux qu'ils seront amenés à faire en dépend. $»^{1}$

\section{NOTES}

1. Her Majesty's Inspectorate, The Curriculum from 5 to 16, $2^{\mathrm{e}}$ edition, HMSO Londres, 1989, p. 26. 


\section{RÉSUMÉS}

Les comparaisons lexicales entre les termes qui désignent l'éthique et la morale dans différentes langues reflètent des approches nuancées des principes et règles de conduite. Parallèlement, le rôle confié à l'école dans ce domaine et les modalités d'un éventuel enseignement de l'éthique sont traités en fonction des dimensions historiques et culturelles propres à chaque pays.

\section{INDEX}

Index géographique : Europe, Espagne, France, Allemagne, États-Unis, Angleterre, Suède, Russie Mots-clés : éducation morale, éthique, système éducatif, éducation

\section{AUTEURS}

\section{CHARLES BALLARIN}

Professeur d'anglais, consultant au CIEP, France

\section{EUGÉNIE ERREA}

Professeur d'espagnol, consultant au CIEP, France

\section{MARTINE SOUQUÈS}

Professeur de russe, consultant au CIEP, France

\section{JOCELYNE THÉBAULT}

Professeur d'anglais, consultant au CIEP, France 